\title{
Development of a novel protein multi-blotting device
}

\author{
Amin M. Hagyousif ${ }^{1}$, Voon J. Chong ${ }^{2}$, Hiroki Yokota ${ }^{3}$, Stanley Y. P. Chien ${ }^{1}$ \\ ${ }^{1}$ Department of Electrical and Computer Engineering, Indiana University Purdue University Indianapolis, Indianapolis, USA; \\ ${ }^{2}$ Department of Biomedical Engineering, Indiana University Purdue University Indianapolis, Indianapolis, USA; \\ ${ }^{3}$ Department of Biomedical Engineering, Rensselaer Polytechnic Institute, New York, USA \\ Email: schien@iupui.edu
}

Received 10 Octorber 2010; revised 18 Octorber 2010; accepted 20 Octorber 2010.

\begin{abstract}
Blotting is a common technique widely used for molecular analysis in life sciences. The Western blot, in particular, is a process of transferring protein samples from a polyacrylamide gel to a blotting membrane and detecting the levels of specific proteins through reactions with primary and secondary antibodies. The state-of-the-art of Western blotting usually generates one blotting membrane per gel. However, multiple copies of blots are useful in many applications. Two blotting copies from a single protein gel, for instance, can be used for identifying a total amount of proteins of interest as well as its specific subpopulation level such as a phosphorylated isoform. To achieve this multi-blotting operation from a single gel, we modified a blotting procedure and developed a novel blotting device. The device consisted of a multi-anode plate and a microcontroller. It was designed to generate a well-controlled electrophoretic voltage profile, which allowed a quasiuniform transfer of proteins of any size. The prototype device was built and its operation procedure was described. The experimental results clearly supported the notion that the described device was able to achieve multiple blotting from a single gel and reduce time and cost for protein analysis.
\end{abstract}

Keywords: Western Blotting; Protein Transfer; Multi-Blotting; PWM

\section{INTRODUCTION}

For detecting the amounts of specific proteins in various biological samples, the Western blot is commonly used among life scientists [1]. This blotting technique utilizes gel electrophoresis to fractionate native or denatured proteins based on their migration speed (mobility) in a gel under an electrical field. The proteins, trapped and size-fractionated in a gel, are transferred and immobi- lized to a positively-charged membrane [2-4]. Using primary antibodies specific to the target proteins as well as secondary antibodies for signal amplification and visualization, expression and modification of the target proteins can be investigated. Although the procedure is well established and many tools are available for gel fractionation and blotting, one of the bottlenecks is its limited efficiency and controllability in the blotting procedure.

In most applications of Western blotting, there is a need to evaluate expression levels of multiple protein targets. Signal transduction pathways, for instance, are often activated by protein modifications such as glycosylation and phosphorylation [5,6]. It is important to determine the amount of signaling molecules that are, in many cases, phosphorylated. However, the current blotting technique can generate only a single membrane per gel and thus life scientists usually have to prepare multiple gels. When the results from multiple gels are compared, there is a concern about potential variations among gels. Furthermore, since the preparation of protein samples, running gels, and transferring to membranes are time consuming and costly, it is desirable to develop a blotting procedure that enables multiple blotting from a single gel. Such a procedure can reduce potential inconsistencies during sample loading, electrophoresis, and blotting, which are affected by varying factors including operational time, temperature, and chemical compositions.

A specific aim of this study was to develop a novel protein blotting device with two goals. First, the blotting system would be able to generate three blotting membrane per gel with equal quality (multi-blotting). Second, it would allow transferring proteins with varying sizes (for instance, 20 to $150 \mathrm{kD}$ ) in the same percentage rate in the same blotting procedure without altering transferring time (uniform transferring).

In order to satisfy the above design goals, we first evaluated efficiency of protein transport by establishing 
a cumulative signal intensity function, $P(t)$, as a function of transferring time, $t$. For instance, $P\left(t_{1}\right)=1 / 3$ implies that one third of proteins is transferred for a duration of $t_{1}$. We then derived an electrophoretic voltage profile along the direction of protein migration, in which one third of proteins regardless of their molecular size would be transferred in $t_{1}$. This voltage profile was applied to a multi-anode plate using a microcontroller based pulse width modulated (PWM) voltage generator [7].

\section{MATERIALS AND METHODS}

\subsection{Western Blotting}

\subsubsection{Protein Samples}

MC3T3 osteoblast-like cells were cultured in MEM medium containing $10 \%$ fetal bovine serum and antibiotics (50 units/ml penicillin and $50 \mu \mathrm{g} / \mathrm{ml}$ streptomycin; Invitrogen) [8]. Cells were incubated at $37^{\circ} \mathrm{C}$ in a humid chamber with $5 \% \mathrm{CO}_{2}$ and prepared for experiments at $70-80 \%$ confluency. Protein samples were isolated by sonicating cells using a sonic dismembrator (Model 100, Fisher Scientific) and lysed in a RIPA lysis buffer [9].

\subsubsection{Electrophoresis and Semi-Dry Transferring}

Isolated proteins were size-fractionated using 10\% SDS gels (1.5 mm thickness). Electrophoresis was conducted using $100 \mathrm{~V}$ for $10 \mathrm{~min}$ (stacking), followed by $150 \mathrm{~V}$ for various durations (separation). Proteins, immobilized in SDS gels, were electro-transferred to Immobilon-P membranes (Millipore) using a semi-dry transferring apparatus (BioRad). The standard transferring condition was $15 \mathrm{~V}$ for $40 \mathrm{~min}$. To evaluate the effects of transferring conditions such as voltages and running times, varying transferring conditions were also examined.

\subsubsection{Antibody Reactions and Image Analysis}

The Immobilon-P membrane after protein blotting was incubated overnight in a blocking solution $(1 \%$ milk in a PBS buffer). The membrane was then incubated for 1 hour with monoclonal $\beta$-actin antibodies (Sigma) followed by $45 \mathrm{~min}$ incubation with goat anti-mouse $\mathrm{IgG}$ conjugated horseradish peroxidase (Amersham Biosciences). The protein levels were assayed using an ECL Western blotting detection kit (Amersham Biosciences), and signal intensities were quantified using a luminescent image analyzer (LAS-3000, Fuji Film) [10].

\subsection{Protein Multi-Blotting Device}

\subsubsection{Prediction of the Blotting Voltage Profile}

In order to uniformly transfer proteins regardless of their size in given transferring time, our approach was to regulate blotting voltages for individual proteins. Based on well-known observation in size fractionation, we assumed that the mobility of proteins was proportional to an applied electrophoretic voltage and transferring time.
Through experimentation, we predicted the blotting voltage profile along the direction of protein migration to achieve a uniform transfer of proteins of any size. In the described blotting device, a protein with a normalized mobility of $r$ would receive a local blotting voltage that was inversely proportional to $r$ (Appendix A).

\subsubsection{Design of the Multiple Anodes and the Microcontroller Based PWM Generator}

The described multi-blotting device has a plate with multiple anodes (Figure 1). With a properly regulated blotting voltage profile, proteins of the identical molecular size would receive the same voltage, while larger proteins would be transferred with the higher voltage. Here, we used a single DC voltage source and a computer controlled voltage generator to provide voltages for all anodes (Figure 2).

This generator was designed to provide multiple pulse width modulated (PWM) DC signals of different duty cycles for all anodes, in which each PWM signal was individually amplified by a PWM amplifier and applied to one anode. The voltage amplification gains are the same to all PWM signals. The duty cycle was set to make the average voltage of an amplified PWM equal to

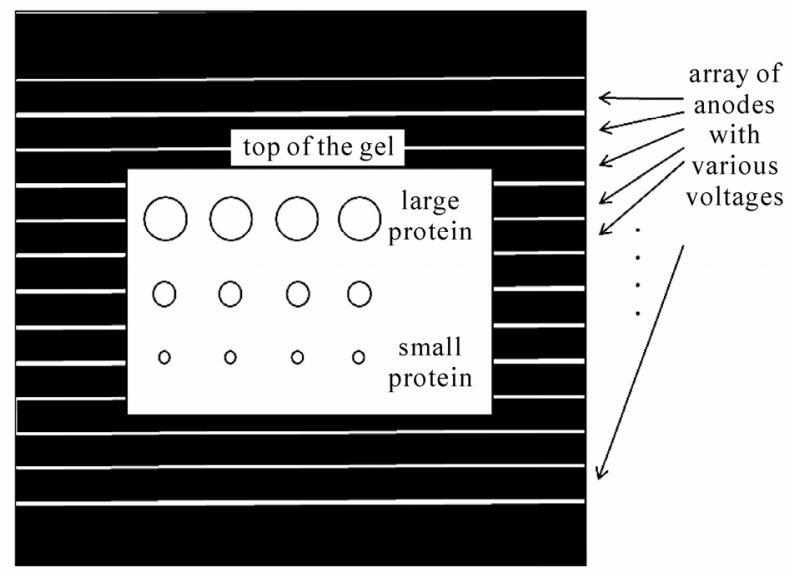

Figure 1. Schematic illustration of the multi-anode plate with a gel. The black stripes designate an array of anodes, and the gel contains size-fractionated proteins (white circles - not in scale).The top anodes receive higher voltage than the bottom anodes.

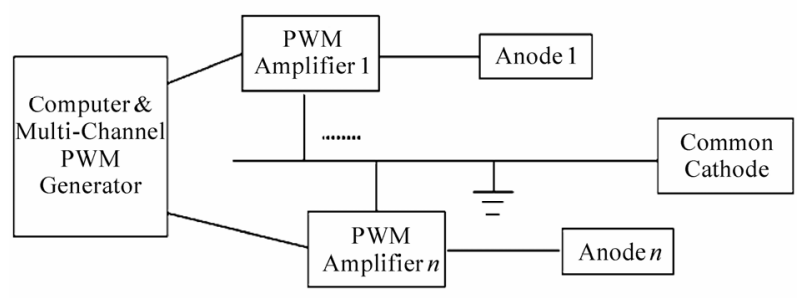

Figure 2. Systems block diagram for PWM voltage control in multi blotting, in which " $n$ " designates the number of anodes. 
the desired driving voltage of the corresponding anode. Assuming the amplification gain is $G$, the peak voltage of all PWM after amplification is $V$, and the desired the voltage for the anode $i$ is $v_{i}$, the duty cycle of the voltage applied to anode $i$ is $\left[v_{i} /(V / G)\right]^{*} 100 \%$.

\section{RESULTS}

\subsection{Experimental Evaluation and Validation}

\subsubsection{Blotting Time and Signal Intensities}

Prior to generating multiple blotting membranes from a single gel, we first conducted semi-dry blotting using various transferring times and built a relationship of signal intensities to transferring time. Using $\beta$-actin as an example, representative blotting images are shown for blotting for $5,13,20,40,60,90$, and $120 \mathrm{~min}$ at $15 \mathrm{~V}$ (Figure 3(a)). Note that two protein bands in each image were generated using the identical conditions. The signal level after reactions with antibodies was initially increased as the blotting time was lengthened. However, the level was saturated for the transfers for $40 \mathrm{~min}$ or longer durations.

The normalized signal intensities showed that this temporal signal profile was approximated by a cumulative Gaussian distribution function (mean $=22 \mathrm{~min}$, and standard deviation $=9 \mathrm{~min})($ Figure $3(\mathbf{b}))$. This cumulative distribution function provided the basis for determining transferring time for each of the multiple blotting membranes. Note that if this cumulative distribution function is a linear function of transferring time, the same blotting time is applied to all membranes. The Gaussian distribution implies that for generating 3 membranes, the $1^{\text {st }}$ and the $3^{\text {rd }}$ transferring durations are identical. The $2^{\text {nd }}$ transferring duration is calculated $83 \%$ of the standard deviation (approximately $7.5 \mathrm{~min}$ in our experimental setup).

\subsubsection{Multiple Transfers}

Based on the relationship between blotting time and signal intensity in the previous experiment, we next conducted multiple transfers (generation of 3 blotting membranes) using a single gel. Focusing on $\beta$-actin, the transfer time was chosen to be $15 \mathrm{~min}$ ( $1^{\text {st }}$ transfer $), 7$ min ( $2^{\text {nd }}$ transfer $)$, and $15 \mathrm{~min}$ ( $3^{\text {rd }}$ transfer $)$. The result clearly shows that by properly choosing blotting durations it is possible to generate multiple blotting membranes, although there are variations in signal intensity among three membranes (Figure 4(a)). In each image, two protein bands were processed using the same experimental condition.

\subsubsection{Transfer under a Non-Uniform Voltage Profile}

The experiments above support our first design goal for achieving multiple blotting membranes from a single gel. To examine the second design goal of simultaneously
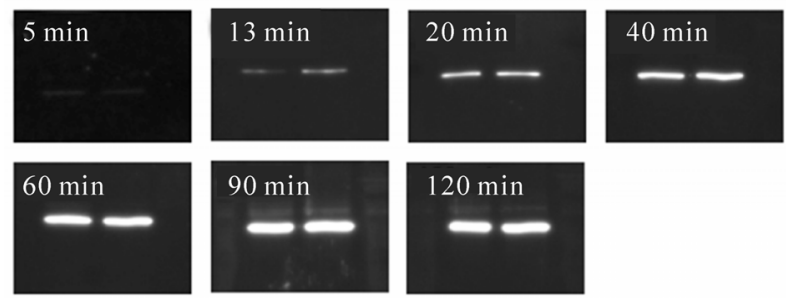

(a)

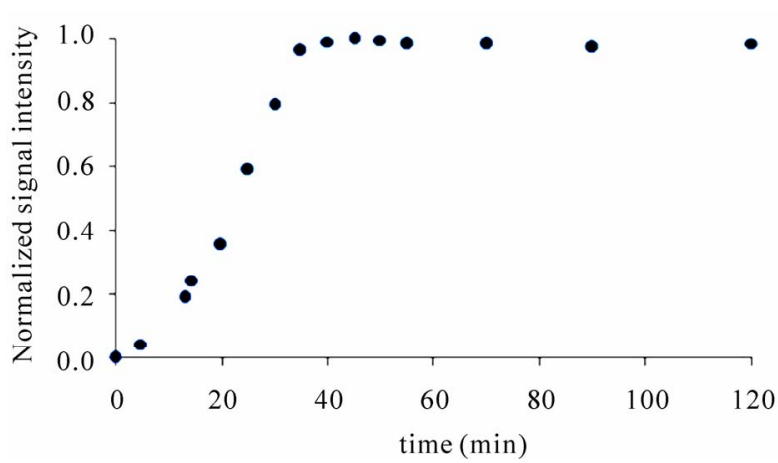

(b)

Figure 3. Signal intensities of $\beta$-actin with various blotting durations. (a) Representative blotting images after semi-dry transferring for 5, 13, 20, 40, 60, 90, and 120 min. (b) Normalized signal intensities as a function of 15 blotting durations. The maximum intensity is set to 1 .
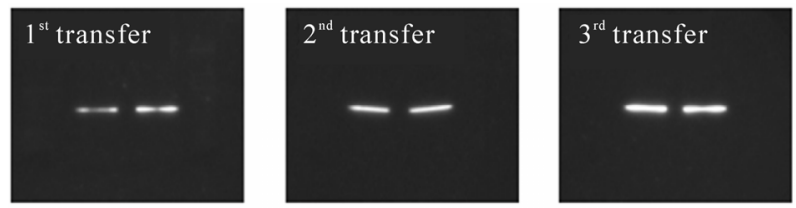

(a)

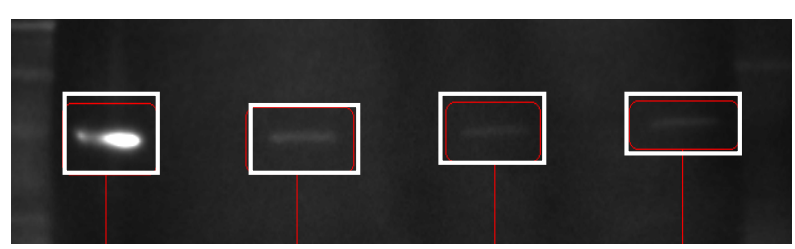

(b)

Figure 4. Multiple transfers and a transfer using a non-uniform voltage profile. (a) Blotting images of three transfers. The transfer time was $14.5 \mathrm{~min}$ ( $1^{\text {st }}$ transfer $), 6.5 \mathrm{~min}$ ( $2^{\text {nd }}$ transfer $)$, and $15 \mathrm{~min}$ ( $3^{\text {rd }}$ transfer). (b) Differential transfer efficiency using a non-uniform voltage profile. The white boxes indicate the expected positions of $\beta$-actin bands, in which 4 different voltages are employed $(12.0,3.9,2.3$, and $1.7 \mathrm{~V}$ from left to right).

transferring proteins with various sizes, we conducted 40-min semi-dry blotting using a non-uniform voltage profile (Figure 4(b)). In this experiment all conditions were identical to the condition employed for generating 
the results depicted in Figure 3 except the voltages. The voltages applied to four different bands (left to right) were $12.0 \mathrm{~V}, 3.9 \mathrm{~V}, 2.3 \mathrm{~V}$, and $1.7 \mathrm{~V}$, respectively. The result using $\beta$-actin as an example demonstrates that transfer efficiency is controllable by an applied electrophoretic voltage. The left position under $12 \mathrm{~V}$ exhibits a clear protein band, but the right position under approximately $1.7 \mathrm{~V}$ barely shows a detectable protein band.

\subsection{Protein Multi-Blotting Device}

\subsubsection{Design and Prototyping the Multi-Anode Plate}

To demonstrate the feasibility of our design principle, a prototype device was made. A computer printed circuit board was etched to make a multiple anodes plate, where the width of each anode stripe was set to be $4 \mathrm{~mm}$ and the width of the gap between two anodes $1 \mathrm{~mm}$ (Figure $5)$. The size of both anode and cathode plates were 18 $\mathrm{cm} \times 24 \mathrm{~cm}$, and the prototype device was designed to accommodate gels with size up to $14 \mathrm{~cm} \times 17 \mathrm{~cm}$.

\subsubsection{Design and Prototyping Multi-Blotting Control Circuits}

A PICF184515 microcontroller-based voltage generator was designed and prototyped to provide the designated voltage profile to individual anodes (Figure 6). This microcontroller generated PWM voltage signals were

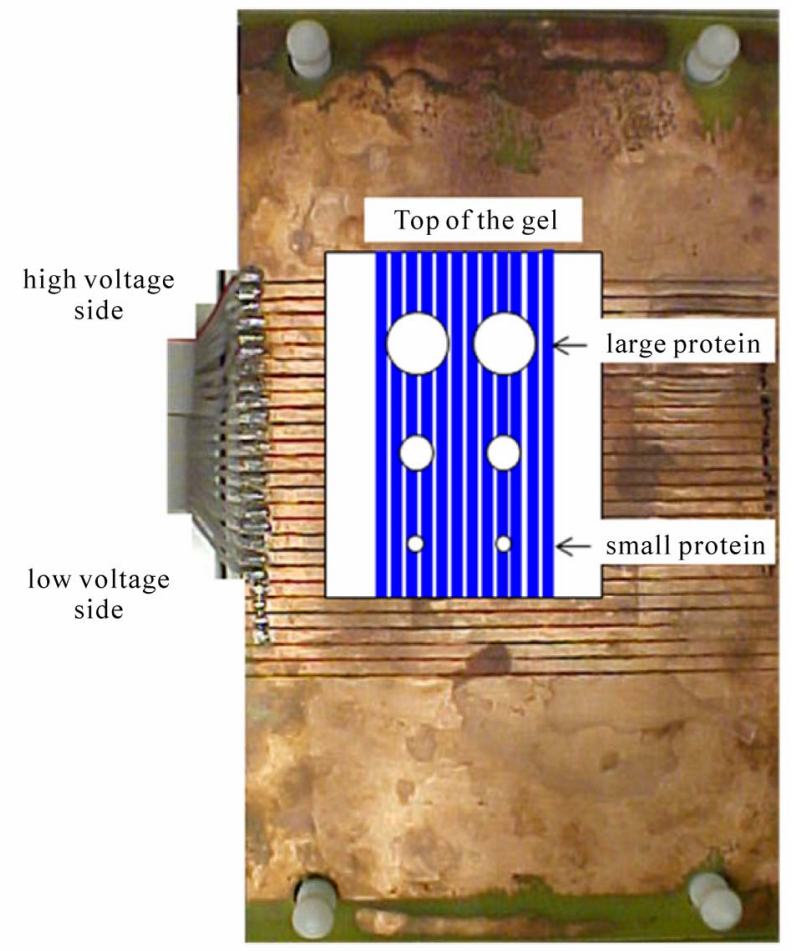

Figure 5. Prototype of a multi-anode plate. The vertical, parallel blue lines indicate the direction of protein migration. The anodes are arranged horizontally, and the top arrays receive higher voltage than the bottom arrays. amplified (Figure 7). The average voltage of the amplified PWM signal corresponded to the desired voltage for the anode.

The maximum voltage of all PWM signals, generated by the microcontroller, was $5 \mathrm{~V}$ with the current of $100 \mu \mathrm{A}$. Since the PWM signals did not provide the necessary power for driving a protein transfer, these signals were amplified using a TC 4469 MOSFETS amplifier. The maximum voltages of all PWM signals were set to $15 \mathrm{~V}$. Figure 8 shows the photograph of the prototype.

During blotting, the microcontroller device was designed to request a user to provide the conditions used for protein fractionation with the vertical gel as well as the conditions for protein blotting. The former fractionation conditions included length and thickness of the gel, electrophoretic voltage, and running time. The latter transferring conditions were the power supply voltage, the number of membranes to be blotted, and buffer solution type used in horizontal blotting and vertical gel. The program in the microcontroller calculated the voltage levels needed for each anode and the total time required for transferring proteins. Based on the mean and the s.d. values of Gaussian distributions, the blotting time required for each membrane was determined. The program gave a sequence of instructions and guide to a user such as "place the membrane on the device," "start blotting," "wait," "change the membrane," etc.

\section{DISCUSSION}

We described a novel protein multi-blotting device consisting of the multi-anode plate and the microcontroller-based voltage-regulating circuits. In experimental evaluations, we modeled blotting signal intensities as a cumulative Gaussian distribution function of transferring durations and demonstrated that it was possible to generate 3 blotting membranes from a single gel. In the device development phase, an appropriate electrophoretic voltage profile was derived and implemented using the multi-anode plate and the microcontroller based PWM voltage generator. The results using $\beta$-actin as an example supported the notion that the described device was capable of providing superior quality for comparing the level of various proteins, reducing the required amount of samples, time, and cost for protein blotting.

In generating a transferring voltage profile, we assumed that protein mobility was proportional to applied voltage and transferring time. In order to regulate differential mobility of proteins with varying sizes, the new multi-blotting device generated a well-controlled voltage profile in which individual proteins received transferring voltage inversely proportional to its intrinsic mobility. Although we presented this particular design with multianodes, it would be possible to build a device using 


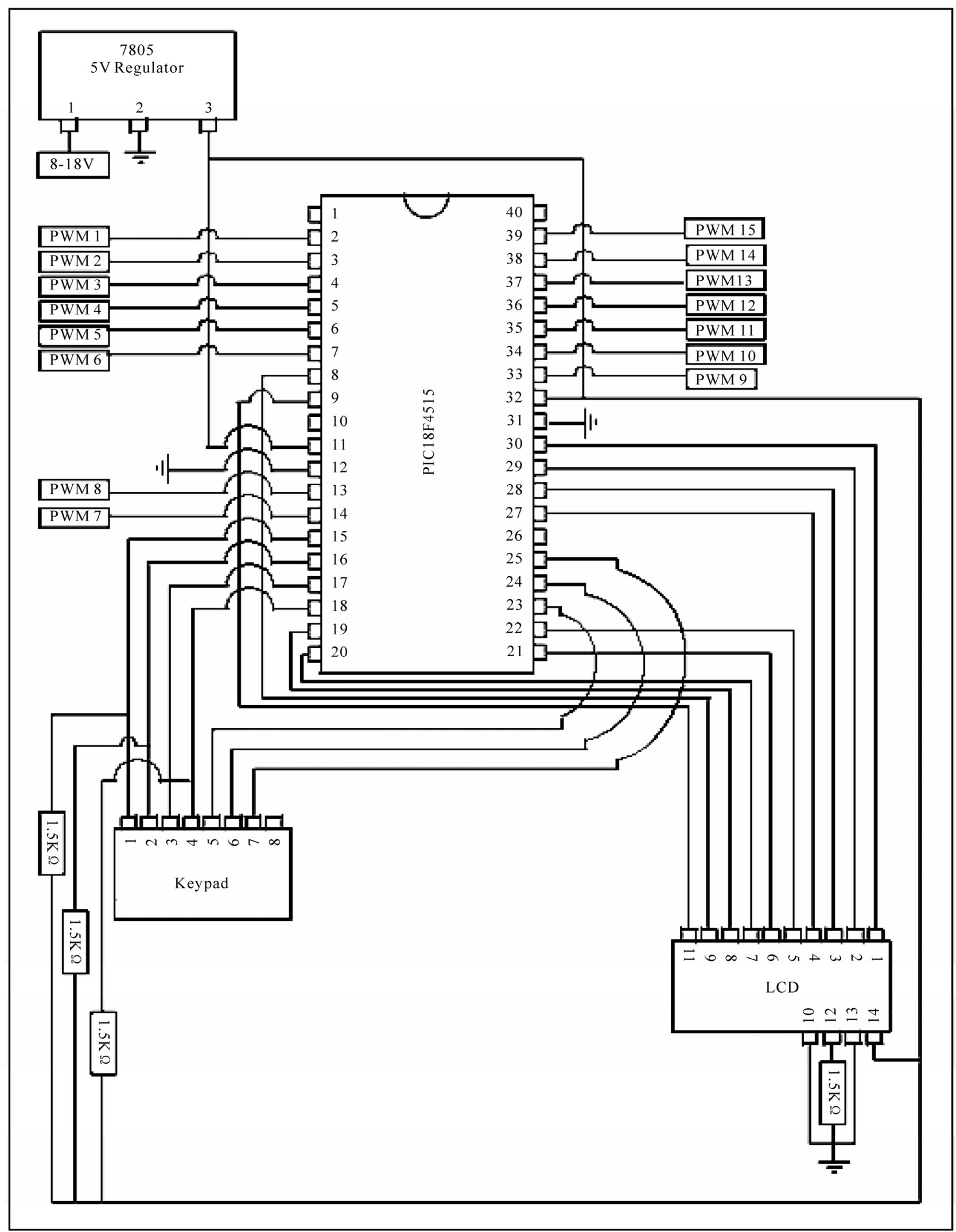

Figure 6. Microcontroller circuits for generating a series of PWM signals for establishing a well-controlled voltage profile to an array of anodes. 

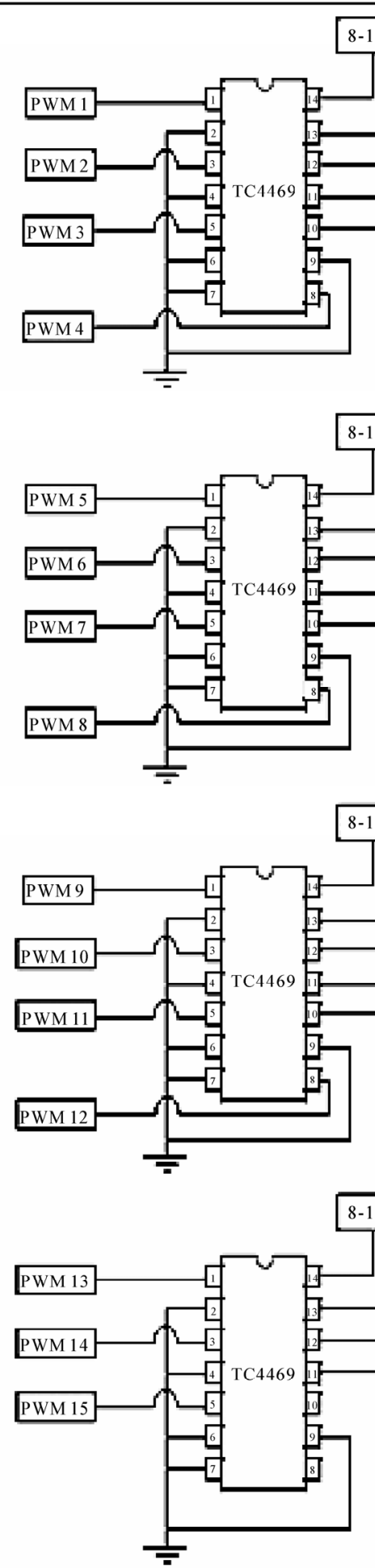

\section{$8-18 \mathrm{~V}$}

$8-18 \mathrm{~V}$
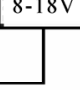


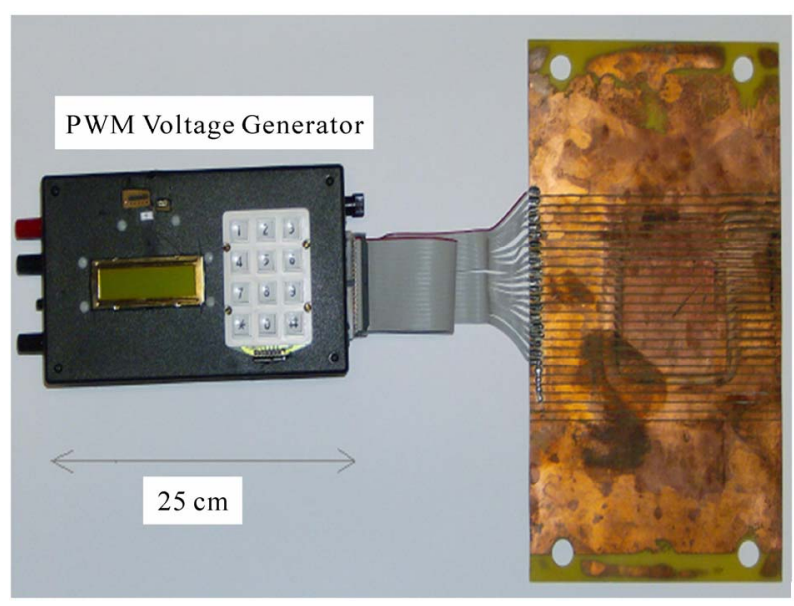

Figure 8. Prototype device for multi bloting. The photograph shows the multi anode plate and the microcontroller based PWM voltage generator.

multi-cathodes and a common anode.

In predicting transferring time for each of the multiple membranes, we built a protein mobility model using a Gaussian function. Although average mobility can be characterized by blotting voltage and running time, any proteins of interest with same size present a variation in their mobility. Based on our experimental result, this variation was approximated by a Gaussian distribution and the amount of proteins transferred to a membrane was predicted from a cumulative Gaussian distribution. By determining the mean and s.d. values, it was straightforward to divide the transfer process into multiple segments, each of which would receive the equal amount of proteins of interest.

In our initial prototype design, copper was used as material for the anode and stainless steel for the cathode. We observed that electrical platting occurred and a noticeable amount of cooper was transferred along with proteins to the cathode. Other materials such as stainless steel or platinum-titanium alloy should be used to avoid the observed electrical platting during the blotting process.

In predicting and evaluating the capability of the device, we employed commonly used materials and conditions such as $10 \%$ SDS gel, a transfer buffer with $70 \%$ methanol, etc. Fine tuning is necessary in determining the voltage profile and running time when other materials and conditions are used. Although we focused on the operation of the described device for semi-dry blotting, the same principle should apply to dry blotting.

\section{CONCLUSION}

This paper described the novel multi-blotting device that enabled transferring proteins of any sizes to multiple blotting membranes from a single gel. Two design goals were multiple blotting and a quasi-uniform transfer of most proteins in $20-150 \mathrm{kD}$. These goals were achieved by the selection of transferring time for each of the multiple blotting membranes, and the alteration of blotting voltages for individual proteins with different sizes. The device was designed and prototyped using the multianode plate and the microcontroller based voltage generator, and a well-controlled voltage profile was generated.

\section{ACKNOWLEDGEMENTS}

The authors appreciated technical support of Chang Jiang.

\section{REFERENCES}

[1] Towbin, H., Staehelin, T. and Gordon, J. (1979) Electrophoretic transfer of proteins from polyacrylamide gels to nitrocellulose sheets procedure and some applications. Proceedings of the National Academy of Sciences (USA), 76, 4350-4354.

[2] Chen, H. (2003) Semi-dry electroblotter. US Patent $6,592,734 \mathrm{~B} 2$

[3] Adcock, M. (1990) Field inversion elecroblotting \& electroElution. US Patent 4959133.

[4] Zahringer, H. (2008) Product survey: Blotting equipment, low-tech at its best. lab times, May, 55-59.

[5] Ajit, V. (2009) Essentials of glycobiology. 2nd Edition, Cold Spring Harbor Laboratories Press.

[6] Tarrant, M.K. and Cole, P.A. (2009) The chemical biology of protein phosphorylation. Annual Review of Biochemistry, 78, 797-825.

[7] Quercioli, V. (1993) Pulse width modulated (PWM) power supplies, Vol. 45(Studies in Electrical and Electronic Engineering). Elsevier, Amsterdam.

[8] Wang, D., Christensen, K., Chawla, K., Xiao, G., Krebsbach, P.H., and Franceschi, R.T. (1999) Isolation and characterization of MC3T3-E1 preosteoblastsubclones with distinct in vitro and in vivo differentiation/mineralization potential. Journal of Bone Mineral Research, 14, 893-903.

[9] Hirasawa, H., Jiang, C., Zhang, P., Yang, F.C. and Yokota, H. (2010) Mechanical stimulation suppresses phosphorylation of eIF2 and PERK-mediated responses to stress to the endoplasmic reticulum. FEBS Letters, 584, 745-752.

[10] Hamamura, K. and Yokota, H. (2007) Stress to endoplasmic reticulum of mouse osteoblasts induces apoptosis and transcriptional activation for bone remodeling. FEBS Letters, 581, 1769-1774. 


\section{APPENDIX}

For a protein transferring in a gel, its moving distance, $d$, can be modeled to be proportional to the electrophoretic voltage, $v$, and blotting time, $t$ :

$$
d=k v t
$$

with $k$ as a proportional factor. Suppose that for a fixed time, $T$, a moving distance is expressed as $d=r L$, where $L=$ length of the gel, and $r=$ normalized mobility ratio between 0 (no mobility) and 1 (maximum mobility). In the described apparatus, any proteins have the same mo- bility along the thickness of the gel for a constant time with a graded electrophoretic voltage for individual proteins. Assume that a largest protein of interest moves $d_{\text {min }}$ at $v_{\max }$ with $d_{\min }=r_{\min } L$. Then, $v$ for any protein with a normalized mobility ratio of $r$ can be determined:

$$
\mathrm{v}=v_{\max } *\left(r_{\min } / r\right)
$$

In summary, the graded electrophoretic voltage in the described apparatus is regulated to be inversely proportional to its normalized mobility ratio. 